\title{
Problem poser-how to interpret divergent prognostic evidence of simultaneously measured tumor markers?
}

\author{
U. KLINGE $\uparrow * *$ E. DAHLף and P. R. MERTENS§
}

\begin{abstract}
$\dagger$ Applied Medical Engineering, Helmholtz Institute, RWTH Aachen University, Aachen, Germany
\$Surgical Department, University Hospital of the RWTH Aachen, Aachen, Germany

I Institute of Pathology, University Hospital of the RWTH Aachen, Aachen, Germany

$\S$ Department of Nephrology and Clinical Immunology, University Hospital of the RWTH Aachen, Aachen, Germany
\end{abstract}

(Received 2 February 2007; revised 12 February 2007; in final form 23 February 2007)

In the past, even complex systems have been regarded mainly as a collection of components which work together in a definable way like a technical machine. The clear functional connection between two components (nodes) A and B underlines a direct causal relationship and allows the prediction of the effect for B after acting on A. In contrast, current consideration of biological systems increasingly includes the many cross-links and feedback loops that form a functional network. Correspondingly, in a network it is more difficult to predict the response of B to any stimulating effect applied to A, and thereby to verify the causal impact.

Generally, a highly interconnected network is hard to control, but a system without any crosslinking is more susceptible to loss of function, due to any interruption [1,2]. The construction of many current networks, with only few nodes with low degree of crosslinking and many nodes that are highly linked, appear to be the most favourable to guarantee both resistance to damage and maintained ability to differential regulation. However, such a construction, which has developed to become highly effective during evolution, raises questions for our common thinking of causal relationships and causal relevance.

Although human beings should be regarded as a biological system rather than a machine, in medicine, it is often preferable to assume direct functional and causal relationships. For various malignancies, tissue-specific tumour markers have been identified, which indicate a low or high risk for recurrence of tumour growth. Their usefulness is tested by relating the relapses to the expression of these markers. Whereas estrogen- and progesterone-receptor expressions are assumed to be favourable for the patient [3,4] because they reflect a more differentiated (and hence less aggressive) tumour, the proto-oncogene $p 53[5,6]$ and human epidermal growth factor receptor (HER2) [7] are associated with accelerated malignant growth. Additionally, the nuclear Y-box (YB) protein-1 staining pattern in breast cancer cells and surrounding tissue has been shown to be predictive for drug resistance, indicating a poorer long-term outcome [8]. Hence, in general it is assumed that expressions of estrogen- or

*Corresponding author. Email: klinge@hia.rwth-aachen.de 
progesterone-receptor may be favourable whereas the expressions of HER2-receptor, p53 or YB-1 may be indicative of a more aggressive tumour phenotype.

The expression of five different prognostic markers have been analysed in a group of 178 breast cancer patients with breast cancer. The result of this analysis was intriguing, because for most patients the different markers gave divergent prognostic evidence. Only 3 of 129 patients revealed a uniform constellation of low risk with positive estrogen- and progesterone-receptor in accordance with negative staining for p53, HER2-receptor and YB-1, whereas only 15 patients revealed a uniform constellation of poor prognosis with negative estrogen and progesterone receptor and positive staining for p53, HER2-receptor and YB-1. Coding each marker with " 0 " and " 1 " results in a figure of five digits with a total of 32 possible combinations. However, in fact only 24 of the possible 32 combinations have been realized in this cohort (table 1).

If the markers were completely independent of recurrence status and from each other, an equal expression in $3 \%$ of the patients for each combination should be expected. In contrast, we saw a distinct distribution, depicted in table 1, with a most frequent combination of "10001" (estrogen-receptor positive, progesterone- and HER2-receptor as well as p53 negative, YB-1 positive). The regular course of the frequencies following a power law is intriguing and has yet to be clarified. However, the confusing distribution of the marker groupings reduces sensitivity, specificity and correlation coefficients and reflects the limited value of each marker to predict mortality due to recurrence for the entire cohort (table 2).

The question for the clinician is how can a combination of markers lead to enhanced prognostic evidence for the individual patient?

In general there would be no problem for clinical use, if only two markers are measured, even if they show some overlap (figure 1a,b). There is also no problem using five markers, if

Table 1. Combinations of the marker proteins estrogen-, progesterone-, HER2-receptor, p53 and YB-1, sorted according to absolute frequency, with the percentage of being expressed in patients with death for recurrence, coded as staining negative $=0$ and positive $=1$ for estrogen-, progesterone-, HER2-receptor, p53 and YB-1.

\begin{tabular}{lrrr}
\hline Combination of marker proteins & $n$ & Frequency $(\%)$ & Death for recurrent cancer $(\%)$ \\
\hline "10001" & 16 & 12.3 & 50 \\
"11000" & 15 & 11.5 & 13 \\
"10000" & 12 & 9.2 & 84 \\
"10011" & 11 & 8.5 & 20 \\
"11001" & 10 & 7.7 & 0 \\
"00011" & 8 & 6.2 & 0 \\
"00000" & 7 & 5.4 & 29 \\
"11010" & $71011 "$ & 5.4 & 0 \\
"00010" & 6 & 4.6 & 40 \\
"10111" & 5 & 3.9 & 40 \\
"00001" & 5 & 3.9 & 100 \\
"10010" & $50100 "$ & 3.1 & 25 \\
"00101" & 4 & 3.1 & 100 \\
"00111" & 4 & 2.3 & 67 \\
"10110" & 3 & 2.3 & 67 \\
"011000" & 3 & 0 \\
\end{tabular}

Estrogen receptor neg/pos, 0/1; progesterone receptor neg/pos, 0/1; HER2 receptor neg/pos, 0/1; p53 neg/pos, 0/1; YB-1 neg/pos, 0/1. 
Table 2. Sensitivity, specificity and Pearson correlation coefficient for the prediction of death for cancer recurrence of 178 patients with breast cancer.

\begin{tabular}{lccc}
\hline & Sensitivity $(\%)$ & Specificity $(\%)$ & Pearson correlation with recurrence status \\
\hline Estrogen receptor & 42 & 31 & -0.138 \\
Progesterone receptor & 11 & 55 & -0.224 \\
HER2 receptor & 11 & 79 & 0.179 \\
P53 & 22 & 51 & 0.025 \\
YB-1 & 19 & 66 & 0.242 \\
\hline
\end{tabular}

there is no overlap, since each marker may detect some more patients (figure 1c). The problem rises if there are considerable overlaps due to complex interactions between these markers (figure 2). With an increasing number of markers that overlap, rather than increasing confidence, the informative content may even decrease.

The functional connections are reflected by Pearson's correlation coefficients (table 3). The interference with each other via an unknown number of genes and proteins may lead to unpredictable effects. What may be helpful in one situation, may be disadvantageous for other groupings. Though Pearson's correlation coefficient confirmed the general impact of the markers on prognosis, for some individual patients there remains an inconsistent grouping of markers, which simultaneously indicate both a high and a low risk for developing recurrent cancer.

The difficulty for clinicians is how to interpret the divergent prognostic evidence of simultaneously measured tumour markers and what to advise the patient?

There has been a rapid increase of detailed information available for more than 40,000 genes and 100,000 proteins, which all are supposed to be part of a complex network. How can we integrate all the available information to define a concluding and unambiguous result? Does the consideration of multiple interactions mean that in principle we have to use more complex considerations than straight causal relationships between any two components? Although Newton's laws were written in 17th century, it is still challenging to calculate the

(A)

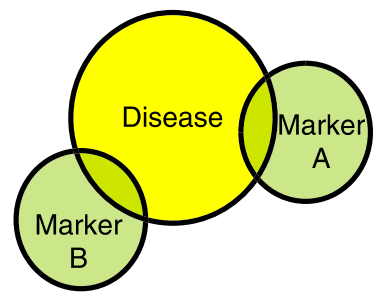

(B)

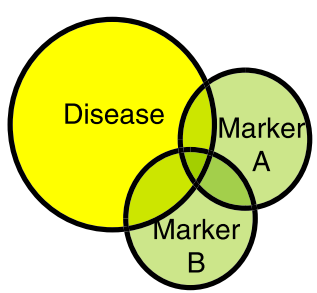

(C)

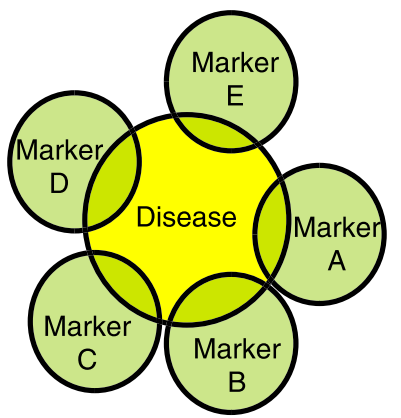

Figure 1. Diagnostic markers to detect a disease: A, two markers without overlap, each adding some patients; B, two markers with overlap, each adding some patients though some are detected by both; C, five markers without overlap, each marker able to add some more patients. 

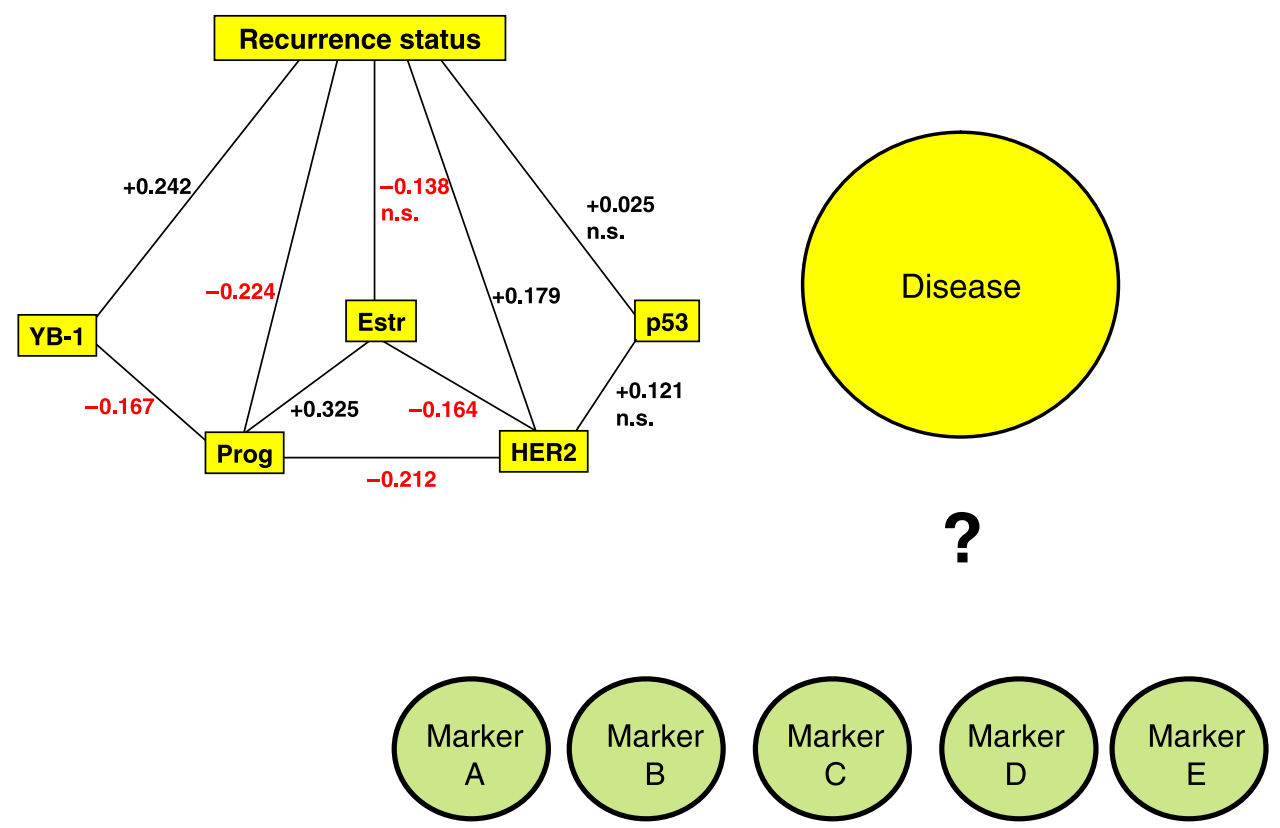

Figure 2. Correlation "network" of tumour markers as risk factor for death because of breast cancer recurrence. Correlations are regarded as significant if $p<0.05$. Each marker adds some patients, but all together for most patients there is a contradictory estimation of recurrence risk. How to connect the markers to disease?

Table 3. Pearson's correlation coefficient for co-expression of tumor markers.

\begin{tabular}{lccccc}
\hline & Estrogen receptor & Progesterone receptor & HER 2 receptor & p53 & YB-1 \\
\hline Estrogen receptor & - & & & & \\
Progesterone receptor & 0.33 & - & & \\
HER 2 receptor & -0.16 & -0.21 & 0.12 & - & \\
P53 & -0.10 & -0.10 & 0.13 & 0.13 & - \\
YB-1 & 0.06 & -0.17 & & \\
\hline
\end{tabular}

gravitational interference of only three planets. In biology and medicine we have to deal with many more components forming a huge network of interactions and interferences. If there are main streams with some few crucial components, which may be suitable as targets for intervention, how can they be found, and how can causal pathways in a network be identified? If correlations indicate functional relationships, is it possible to use the correlations between the components for depicting a map of functional connectivity, which may help to reduce the network to the level of a machine which can then be used to reconstitute causality?

Alternatively, if it is generally impossible to isolate causal pathways within a network, do we need to be more restrictive in our thinking of biology as being merely a simple construct of many causal relationships - a concept used in physics some decades ago to face Heisenberg's uncertainty relations? But then, is there any hope for a quantum theory which can be applied to biology and medicine? 


\section{References}

[1] Jeong, H., Tombor, B., Albert, R., Oltvai, Z.N. and Barabasi, A.L., 2000, The large-scale organization of metabolic networks, Nature, 407(6804), 651-654.

[2] Albert, R., Jeong, H. and Barabasi, A.L., 2000, Error and attack tolerance of complex networks, Nature, 406(6794), 378-382.

[3] Arpino, G., Weiss, H., Lee, A.V., et al., 2005, Estrogen receptor-positive, progesterone receptor-negative breast cancer: association with growth factor receptor expression and tamoxifen resistance, Journal of the National Cancer Institute, 97(17), 1254-1261.

[4] Kute, T.E., Russell, G.B., Zbieranski, N., et al., 2004, Prognostic markers in node-negative breast cancer: a prospective study, Cytometry Part B, Clinical Cytometry, 59(1), 24-31.

[5] Osanai, T., Takagi, Y., Toriya, Y., et al., 2005, Inverse correlation between the expression of O6-methylguanineDNA methyl transferase (MGMT) and p53 in breast cancer, Japanese Journal of Clinical Oncology, 35(3), $121-125$.

[6] Gurkan, A., Erdogan, G., Erdogan, O., et al., 2004, Expression of c-erbB-2 and p53 in breast carcinoma patients: comparison with traditional prognostic factors and survival, The Journal of International Medical Research, 32(5), 455-464.

[7] Pellikainen, J.M., Ropponen, K.M., Kataja, V.V., Kellokoski, J.K., Eskelinen, M.J. and Kosma, V.M., 2004, Expression of matrix metalloproteinase (MMP)-2 and MMP-9 in breast cancer with a special reference to activator protein-2, HER2, and prognosis, Clinical Cancer Research, 10(22), 7621-7628.

[8] Janz, M., Harbeck, N., Dettmar, P., et al., 2002, Y-box factor YB-1 predicts drug resistance and patient outcome in breast cancer independent of clinically relevant tumor biologic factors HER2, uPA and PAI-1, International Journal of Cancer, 97(3), 278-282. 


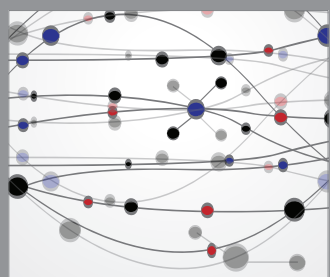

The Scientific World Journal
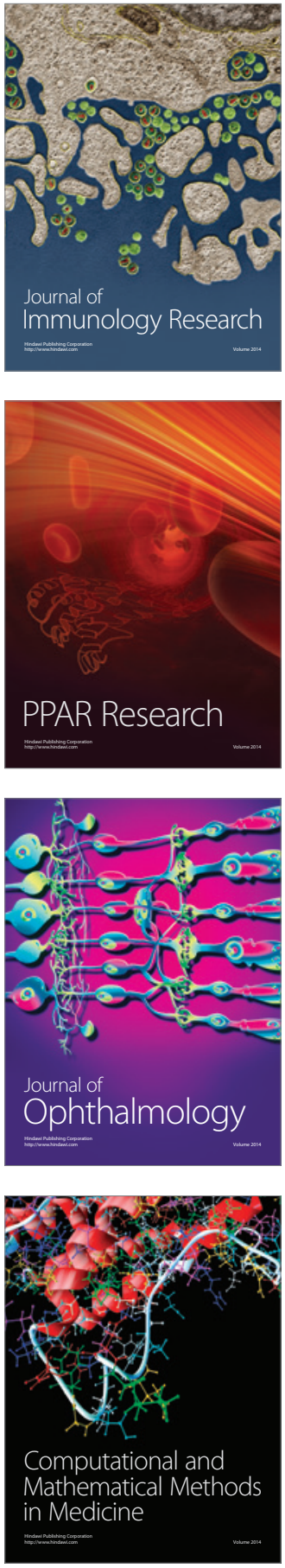

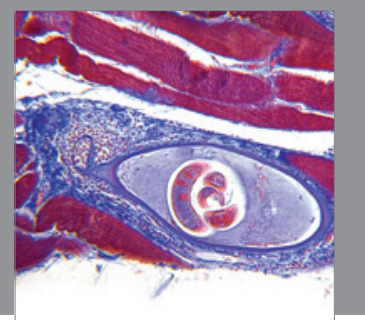

Gastroenterology

Research and Practice
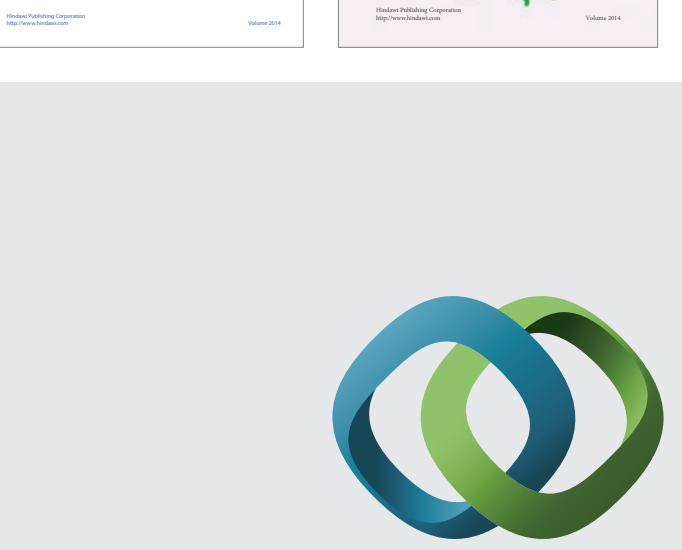

\section{Hindawi}

Submit your manuscripts at

http://www.hindawi.com
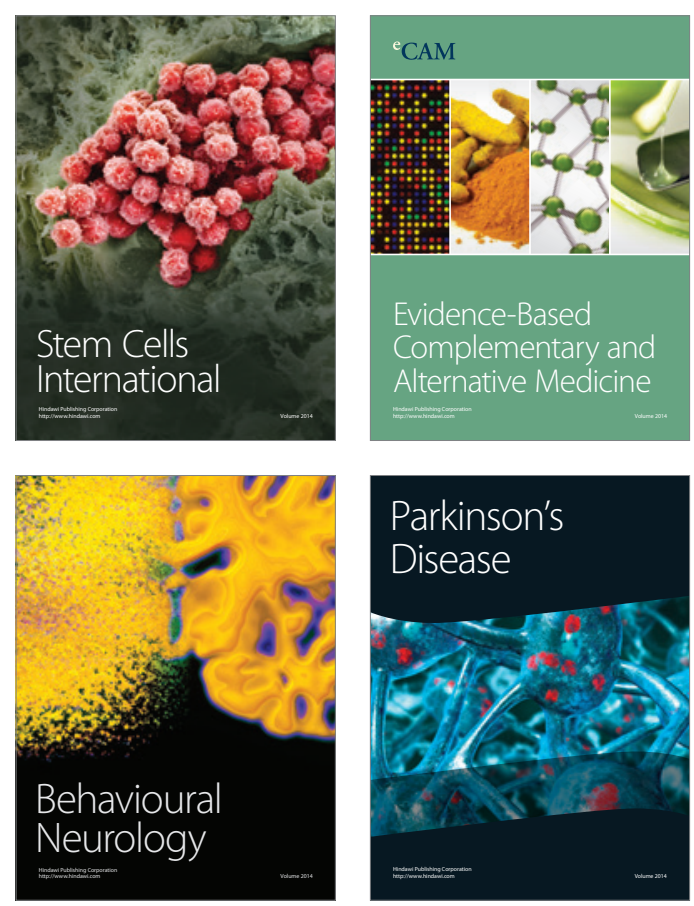

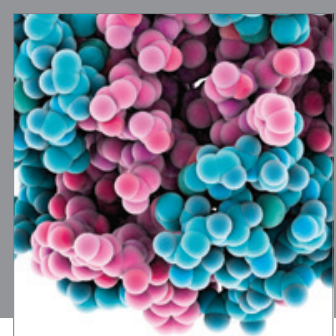

Journal of
Diabetes Research

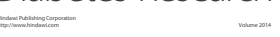

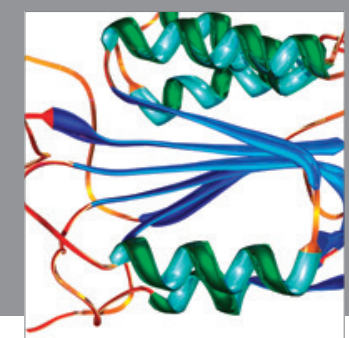

Disease Markers
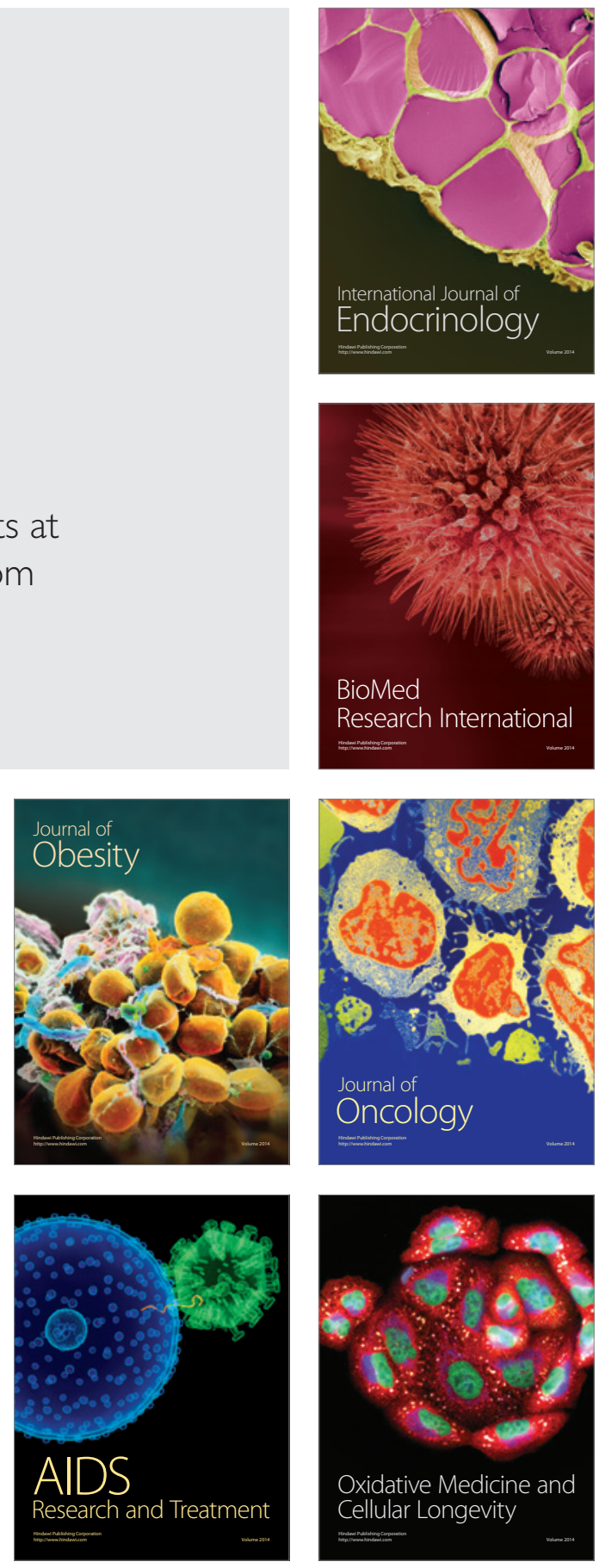